\title{
Plants used as painkillers in folk medicine in Turkey IV - TOOTHACHE
}

\author{
Meryem Șeyda Erbay*, Sezin Anıl, Gülay Melikoğlu \\ Department of Pharmacognosy, Faculty of Pharmacy, Istanbul University, 34116, Istanbul, Turkey.
}

Cite this article as: Erbay MȘ, Anıl S, Melikoğlu G. Plants used as painkillers in folk medicine in Turkey IV - TOOTHACHE. Istanbul J Pharm 48 (2): 49-54.

\begin{abstract}
Turkey has a rich plant diversity due to its geography and many of these plants are used in the treatment of various diseases. The usage of these medicinal plants has been passed down from generation to generation. Ethnobotanical studies are used to record traditional treatment methods and this information is intended to contribute to drug development studies. This study (which is the fourth article in a series focusing on plants with painkilling properties) details the plants used to combat toothache. In this survey, which was prepared by screening ethnobotanical research, a total of 52 taxa were traditionally used in toothache treatment in Turkey. Scientific and local names, families, parts used, and treatment methods of these plants were recorded. According to the results of the research, the most common families are Lamiaceae, Asteraceae, Solanaceae, Euphorbiaceae, Liliaceae and Rosaceae. The most commonly used taxa in different regions of Turkey are Cornus mas, Dianthus zonatus var. zonatus and Hyocyamus niger. These plants contain analgesic and anti-inflammatory compounds. These plants are usually used externally as extracts (infusion/decoction) as well as are used directly. However, a few species are also used externally.
\end{abstract}

Keywords: Toothache, medicinal plants, traditional treatment, Turkey

\section{INTRODUCTION}

Pain is an important health problem that affects most people and develops due to various reasons. Pain has been defined by the International Association for the Study of Pain (IASP) as "an unpleasant sensory and emotional experience associated with actual or potential tissue damage or described in terms of such damage." (Renn and Dorsey 2005). The first step when dealing with a patient experiencing pain is to identify the cause. Whether the pain is due to infection, cancer or any other underlying the disease should be investigated. After the root cause has been identified, both the cause and the resulting pain should be treated (Electronic resource 1).

According to its climate and geographical conditions, Turkey has a rich flora. Approximately eleven thousand plant species, of which three thousand are endemic, grow in Turkey (Güner et al. 2000; Özhatay et al. 2013; Özhatay et al. 2015). Since ancient times, plants have been used by people as food or to resolve health problems. Traditionally used medicinal plants have been recorded in ethnobotanical investigations. These studies are a key source of information for drug research. In this study, plants which have traditionally been used as painkillers in Turkey have been identified by screening ethnobotanical studies. These findings are classified by pain type and published as a series of articles (Erbay et al. 2017; Erbay et al. 2018a; Erbay et al. 2018b). This study, which is the fourth article in a series, deals with the plants used to treat toothache.

Toothache is a condition that everyone has met at least once in their lifetime and is a discomfort that develops due to various reasons. Dental pain is frequently encountered in regular dental practice, and the diagnosis and treatment of primary toothache is not difficult. Nonodontogenic toothache, however, is not routinely studied. Nonodontogenic toothache was categorized into eight groups by primary disorders as follows: 1) myofascial pain referred to tooth/teeth, 2) neuropathic 
toothache, 3) idiopathic toothache, 4) neurovascular toothache, 5) sinus pain referred to tooth/teeth, 6) cardiac pain referred to tooth/teeth, 7) psychogenic toothache or toothache of psychosocial origin, and 8) toothache caused by various other disorders (Yatani et. al. 2014). In these cases, the underlying disease of the toothache should be treated.

Dental pain generally develops in three forms; toothache during or after meals, long-standing toothache, and injuries. A sharp toothache occurs during the meal, or during the first half hour after meals, as food intake builds up in the dental cavities and the acids produced by these foods aggravate the tooth nerves. To relieve toothache, it is first necessary to remove food residues from the tooth surface and while such residues are removed with the help of a toothbrush or dental floss, the toothache persists for a short time.

In the case of progressive tooth decay, the death of the nerve and infection of the tooth causes a swelling around the tooth and in such a situation, it is difficult to relieve toothache. When a painful toothache is experienced, a pain medication or antibiotics recommended by the dentist should be taken to control the infection.

Injuries in the facial region, jawbone and teeth can result from physical trauma. Fractures and cracking may occur in the jawbone or in the teeth as a result of this trauma, and it is even possible that the tooth may become dislodged. In all these cases, dental treatment should be administered by the dentist. (Electronic resource 2).

Herbal remedies may be helpful in relieving pain and inflammation in simple cases. To this end, there are a variety of plants used by people in Turkey for treating toothache.

\section{MATERIAL AND METHODS}

This study was prepared by analysis of theses at the Council of Higher Education Thesis Center and ethnobotanical studies conducted throughout Turkey with selected plants used for treating toothache.

\section{RESULTS AND DISCUSSION}

This study saw a total of 52 taxa traditionally used for treating toothache in Turkey. The details of the plant taxa forming this research (scientific and local names, families, used parts and treatment methods) are given in Table 1.

These plants are mainly from the Lamiaceae (8 taxa), Asteraceae (5 taxa), Solanaceae (4 taxa), Euphorbiaceae (3 taxa), Liliaceae (3 taxa) and Rosaceae (3 taxa) families (Figure 1). The taxa commonly used in different regions of Turkey are Cornus mas Dianthus zonatus var. zonatus and Hyocyamus niger. Plants are usually used externally as a mouthwash, prepared infusion/decoction or applied directly. In addition, decoction and infusion are prepared and used internally.

These plants are used in toothache treatments as they contain analgesic, anesthetic, antimicrobial and anti-inflammatory compounds. For example, Cornus mas has antimicrobial activity because of its major compounds (anthocyanins, flavonoids and iridoids) (Dindaa et al. 2016). Dianthus zonatus var. zonatus and Syzgium aromaticum, known as 'cloves', is commonly used for toothache. It contains antiseptic and anesthetic compounds (especially eugenol). Clove oil is important for toothache treatment (Baytop 1971). Hyocyamus niger has analgesic and anti-inflammatory activity (Beguma et al. 2010). Many other examples exist as well.

Table 1. The plants used in traditional toothache treatments in Turkey

\begin{tabular}{|c|c|c|c|c|c|}
\hline $\begin{array}{l}\text { Botanical } \\
\text { name }\end{array}$ & Family & Local name & Plant part used & $\begin{array}{l}\text { Preparation, } \\
\text { and use }\end{array}$ & Ref. \\
\hline $\begin{array}{l}\text { Achillea } \\
\text { biebersteinii Afan. }\end{array}$ & Asteraceae & $\begin{array}{l}\text { Arı çiçeği, } \\
\text { Erkurtaran, } \\
\text { Sarı çiçek, } \\
\text { Ayvadana, } \\
\text { Sancı çiçeği }\end{array}$ & Aerial part & Inf., Int. & $\begin{array}{l}\text { (Balos and Akan 2007; } \\
\text { Tuzlacı and Doğan 2010) }\end{array}$ \\
\hline $\begin{array}{l}\text { A. millefolium } \\
\text { L. subsp. pannonica } \\
\text { (Scheele) Hayek }\end{array}$ & Asteraceae & $\begin{array}{l}\text { Ayvadana, } \\
\text { Civanperçemi, } \\
\text { Kurpotu, Sporiş }\end{array}$ & Seed & Ext. & (Vural 2008) \\
\hline Allium sativum $\mathrm{L}$. & Liliaceae & Sarımsak & Bulb & $\begin{array}{l}\text { Crushed, } \\
\text { Water, Ext. } \\
\text { +Salt, } \\
\text { Crushed, Ext. }\end{array}$ & $\begin{array}{l}\text { (Erdoğan 2011) } \\
\text { (Özdemir Nath 2016) }\end{array}$ \\
\hline $\begin{array}{l}\text { Anthemis coelopoda } \\
\text { Boiss. var. bourgaei }\end{array}$ & Asteraceae & $\begin{array}{l}\text { Bubacça, } \\
\text { Papatya }\end{array}$ & Flower & Inf., Ext. & (Vural 2008) \\
\hline Cedrus libani A. Rich & Pinaceae & $\begin{array}{l}\text { Katran ağacı, } \\
\text { Sedir }\end{array}$ & Tar & Ext. & (Güneș et al. 2017) \\
\hline Clematis vitalba $\mathrm{L}$. & Ranunculaceae & $\begin{array}{l}\text { Karabağ, } \\
\text { Deli asma, } \\
\text { Diș otu, } \\
\text { Sarmașık, } \\
\text { Akçabağ }\end{array}$ & $\begin{array}{l}\text { Stem bark } \\
\text { Branch }\end{array}$ & $\begin{array}{l}\text { Ext. } \\
\text { Like a cigarette, } \\
\text { Ext. }\end{array}$ & $\begin{array}{l}\text { (Ecevit Genç and } \\
\text { Özhatay 2006) }\end{array}$ \\
\hline
\end{tabular}


Table 1. The plants used in traditional toothache treatments in Turkey (Continued)

\begin{tabular}{|c|c|c|c|c|c|}
\hline $\begin{array}{l}\text { Botanical } \\
\text { name }\end{array}$ & Family & Local name & Plant part used & $\begin{array}{l}\text { Preparation, } \\
\text { and use }\end{array}$ & Ref. \\
\hline $\begin{array}{l}\text { Convolvulus galaticus } \\
\text { Rostan ex Choisy }\end{array}$ & Convolvulaceae & Sarmașık & Flower & Inf., Mouthwash & (Tuzlacı and Doğan 2010) \\
\hline Cornus mas L. & Cornaceae & Kızılcık, Püren & $\begin{array}{l}\text { Branch } \\
\text { Fruit }\end{array}$ & $\begin{array}{l}\text { Burned, } \\
\text { Immersion } \\
\text { in water, Water, } \\
\text { Ext. } \\
\text { Burned, Cinders, } \\
\text { Ext. } \\
\text { Paste, Int. }\end{array}$ & $\begin{array}{l}\text { (Sadıkoğlu and } \\
\text { Alpınar 2001) } \\
\text { (Özdemir Nath 2016) } \\
\text { (Sağıroğlu et al. 2012) }\end{array}$ \\
\hline $\begin{array}{l}\text { Cupressus } \\
\text { sempervirens L. }\end{array}$ & Cupressaceae & $\begin{array}{l}\text { Selvi, Servi, } \\
\text { Yılbașı ağacı }\end{array}$ & Fruit & Dec., Mouthwash & (Sargın et al. 2013) \\
\hline $\begin{array}{l}\text { Daphne mucronata } \\
\text { Royle }\end{array}$ & Thymelaeaceae & Tevri & Branch & Crushed, Ext. & (Mükemre et al. 2015) \\
\hline Datura stramonium L. & Solanaceae & $\begin{array}{l}\text { Deli patpat, } \\
\text { Tatala, } \\
\text { Mandalak }\end{array}$ & Seed & $\begin{array}{l}\text { Dec., Vapor } \\
\text { Dec., Mouthwash }\end{array}$ & $\begin{array}{l}\text { (Altundağ and } \\
\text { Öztürk 2011) } \\
\text { (Tuzlacı and } \\
\text { Alparslan 2007) }\end{array}$ \\
\hline $\begin{array}{l}\text { Dianthus zonatus } \\
\text { Fenzl var. zonatus }\end{array}$ & Caryophyllaceae & $\begin{array}{l}\text { Karanfil, } \\
\text { Deli karanfil }\end{array}$ & $\begin{array}{l}\text { Seed } \\
\text { Flower }\end{array}$ & $\begin{array}{l}\text { Chewed, Ext. } \\
\text { Chewed, Ext. }\end{array}$ & $\begin{array}{l}\text { (Essen 2008; Saday 2009) } \\
\text { (Vural 2008; Kargıŏlu } \\
\text { et al. 2010) }\end{array}$ \\
\hline $\begin{array}{l}\text { Eryngium billardieri } \\
\text { Delar }\end{array}$ & Apiaceae & Tüsü & Root & Crushed, Sap, Ext. & (Mükemre et al. 2015) \\
\hline $\begin{array}{l}\text { Euphorbia } \\
\text { anacampseros } \\
\text { Boiss. var. } \\
\text { anacampseros }\end{array}$ & Euphorbiaceae & Sütleğen & Latex & Ext. & (Șenkardes 2014) \\
\hline E. macroclada Boiss. & Euphorbiaceae & Sütleğen & $\begin{array}{l}\text { Stem and } \\
\text { leaf latex }\end{array}$ & Ext. & (Șenkardes 2014) \\
\hline E. stricta $\mathrm{L}$. & Euphorbiaceae & Sütleğen & Latex & Ext. & (Ugulu 2011) \\
\hline $\begin{array}{l}\text { Ficus carica } \\
\text { L. subsp. carica }\end{array}$ & Moraceae & İncir & Latex & Ext. & (Saraç et al. 2013) \\
\hline Fumaria officinalis L. & Fumariaceae & $\begin{array}{l}\text { Sahtere, } \\
\text { Nuzla otu }\end{array}$ & Aerial part & Dec., Mouthwash & (Sezik et al. 1997) \\
\hline F. vailantii Loisel & Fumariaceae & Şahtere & Aerial part & Dec., Int. & (Özgen et al. 2012) \\
\hline $\begin{array}{l}\text { Helleborus } \\
\text { orientalis Lam. }\end{array}$ & Ranunculaceae & Bohça otu & Root & Crushed, Ext. & (Tuzlacı and Tolon 2000) \\
\hline Hyocyamus niger L. & Solanaceae & $\begin{array}{l}\text { Mankafa otu, } \\
\text { Sağırkulak, } \\
\text { Kurtluca, } \\
\text { Kulak otu, } \\
\text { Dișotu, } \\
\text { Deli batbat }\end{array}$ & $\begin{array}{l}\text { Leaf } \\
\text { Seed }\end{array}$ & $\begin{array}{l}\text { Like a cigarette, } \\
\text { Ext. } \\
\text { Dec., Vapor, Ext., }\end{array}$ & $\begin{array}{l}\text { (Șenkardes 2014) } \\
\text { (Sezik et al. 1997; Bulut } \\
\text { and Tuzlacı 2009; Özgen } \\
\text { et al. 2012) }\end{array}$ \\
\hline H. reticulatus $\mathrm{L}$. & Solanaceae & $\begin{array}{l}\text { Dağ küncüsü, } \\
\text { Künciye, } \\
\text { Koçan }\end{array}$ & Seed & $\begin{array}{l}\text { Like a cigarette, } \\
\text { Ext. }\end{array}$ & $\begin{array}{l}\text { (Özgen et al. 2012; } \\
\text { Șenkardes 2014) }\end{array}$ \\
\hline Laurus nobilis L. & Lauraceae & $\begin{array}{l}\text { Akdeniz defnesi, } \\
\text { Tenel, Defne }\end{array}$ & Leaf & $\begin{array}{l}\text { +Vinegar, Cooked, } \\
\text { Mouthwash }\end{array}$ & (Saraç et al. 2013) \\
\hline Lilium candidum L. & Liliaceae & Zambak & Leaf & Ext. & (Ugulu 2011) \\
\hline Malva sylvestris L. & Malvaceae & $\begin{array}{l}\text { Ebegümeci, } \\
\text { Kabaot, } \\
\text { Develik, } \\
\text { Kedigözü }\end{array}$ & Aerial part & Inf., Mouthwash & (Sargın et al. 2013) \\
\hline Origanum onites $L$. & Lamiaceae & $\begin{array}{l}\text { Kekik, } \\
\text { Eșek kekiği, } \\
\text { Beyaz kekik, } \\
\text { Deli kekik, } \\
\text { Kara kekik, } \\
\text { Taș kekiği }\end{array}$ & Leaf & Chewed & (Polat and Satıl 2012) \\
\hline
\end{tabular}




\section{Table 1. The plants used in traditional toothache treatments in Turkey (Continued)}

\begin{tabular}{|c|c|c|c|c|c|}
\hline $\begin{array}{l}\text { Botanical } \\
\text { name }\end{array}$ & Family & Local name & Plant part used & $\begin{array}{l}\text { Preparation, } \\
\text { and use }\end{array}$ & Ref. \\
\hline $\begin{array}{l}\text { O. vulgare L. subsp. } \\
\text { hirtum (Link) letswaart }\end{array}$ & Lamiaceae & $\begin{array}{l}\text { Kekik, } \\
\text { Kekikotu, } \\
\text { Deli kekik, } \\
\text { Karakekik, } \\
\text { Güve kekiği, } \\
\text { Yer kekiği, } \\
\text { Ak kekik, } \\
\text { Mercan köșk }\end{array}$ & Leaf & Chewed & (Kültür 2007) \\
\hline $\begin{array}{l}\text { 0. vulgare L. subsp. } \\
\text { viride (Boiss.) Hayek }\end{array}$ & Lamiaceae & $\begin{array}{l}\text { Hoș otu, } \\
\text { Kaya kekiği, } \\
\text { Taș kekik, } \\
\text { Sarı kekik, } \\
\text { Yayla kekiği, } \\
\text { Dağ kekiği }\end{array}$ & Aerial part & Inf., Mouthwash & (Sargın et al. 2013) \\
\hline $\begin{array}{l}\text { Ornithogalum } \\
\text { armeniacum Baker }\end{array}$ & Liliaceae & $\begin{array}{l}\text { Köpek soğanı, } \\
\text { Beyaz sümbül, } \\
\text { Yoğurtcuk otu }\end{array}$ & Bulb & Mush, Ext. & (Sargın et al. 2013) \\
\hline Peganum harmala L. & Zygophyllaceae & $\begin{array}{l}\text { Üzerlik, Harmal, } \\
\text { Nazarlık otu }\end{array}$ & Root & Dec. & (Balos and Akan 2007) \\
\hline Pistacia khinjuk Stocks & Anacardiaceae & Gezan, Bıttım & Stem resin & Chewed & (Akan et al. 2008) \\
\hline Platanus orientalis L. & Platanaceae & Çınar & Leaf & Dec., Mouthwash & $\begin{array}{l}\text { (Tuzlacı and } \\
\text { Sadıkoğlu 2007) }\end{array}$ \\
\hline Plumbago europaea L. & Plumbaginaceae & Serkel otu & Root & Sap, Ext. & (Oral 2007) \\
\hline $\begin{array}{l}\text { Populus usbekistanica } \\
\text { Kom. subsp. } \\
\text { usbekistanica }\end{array}$ & Salicaceae & Servi kavağı & Stem bark & A little part, Ext. & (Fujita et al. 1995) \\
\hline Prunus armeniaca L. & Rosaceae & Zerdali & Fruit & $\begin{array}{l}\text { Cooked, Crushed, } \\
\text { Ext. }\end{array}$ & (Mükemre et al. 2015) \\
\hline $\begin{array}{l}\text { P. spinosa L. subsp. } \\
\text { dasyphylla (Schur) }\end{array}$ & Rosaceae & Güvemotu, & Fruit & Dec., Mouthwash & (Tuzlacı and Eryașar \\
\hline Domin. & & Avşarotu & & & Aymaz 2001] \\
\hline $\begin{array}{l}\text { Quercus infectoria } \\
\text { Olivier }\end{array}$ & Fagaceae & $\begin{array}{l}\text { Mazımeșe, } \\
\text { Kasnak, Meșe, } \\
\text { Pelit, Palamut }\end{array}$ & Root cortex & $\begin{array}{l}\text { Crushed, } \\
\text { Mouthwash }\end{array}$ & (Tetik et al. 2013) \\
\hline Q. robur L. subsp. & Fagaceae & Meșe, Meyral & Cortex & Dec., Mouthwash & (Aktan 2011) \\
\hline robur Rhamnus & Rhamnaceae & Kördiken & Gum & Ext. & (Eșen 2008) \\
\hline $\begin{array}{l}\text { (ycioides L. } \\
\text { R. oleoides ) } \\
\text { L. subsp. graecus } \\
\text { (Boiss. \& Ruet.) }\end{array}$ & $\begin{array}{l}\text { Rhamnaceae } \\
\text { Rosaceae }\end{array}$ & $\begin{array}{l}\text { Kördiken } \\
\text { Böğürtlen }\end{array}$ & $\begin{array}{l}\text { Gum } \\
\text { Root }\end{array}$ & $\begin{array}{l}\text { Ext. } \\
\text { Dec., Int. }\end{array}$ & $\begin{array}{l}\text { (Saday } 2009 \\
\text { (Metin 2009) }\end{array}$ \\
\hline \multicolumn{6}{|l|}{ Holmboe } \\
\hline $\begin{array}{l}\text { Salvia limbata } \\
\text { C.A. Mey. }\end{array}$ & Lamiaceae & Kedi kuyruğu & Aerial part & Dec., Mouthwash & (Özgen et al. 2012) \\
\hline $\begin{array}{l}\text { Solanum nigrum } \\
\text { L. subsp. nigrum }\end{array}$ & Solanaceae & $\begin{array}{l}\text { Tilki üzümü, } \\
\text { İt üzümü }\end{array}$ & Leaf and Fruit & Dec., Ext., Vapor & (Balos and Akan 2007) \\
\hline $\begin{array}{l}\text { Syzgium aromaticum } \\
\text { (L.) Merrill. }\end{array}$ & Myrtaceae & Karanfil & Flower & Chewed & (Șahin Yiğit 2014) \\
\hline $\begin{array}{l}\text { Taraxacum stevenii } \\
\text { (Spreng.) DC. }\end{array}$ & Asteraceae & $\begin{array}{l}\text { Çıtlık, Hindibağ, } \\
\text { Keklik otu, } \\
\text { Kıl çiçek }\end{array}$ & Aerial part & Inf., Int. & $\begin{array}{l}\text { (Özdemir and } \\
\text { Alpınar 2015) }\end{array}$ \\
\hline $\begin{array}{l}\text { Teucrium chamaedrys } \\
\text { L. subsp. chamaedrys }\end{array}$ & Lamiaceae & $\begin{array}{l}\text { Bodurca Mahmut, } \\
\text { Tatarca otu, } \\
\text { Sancı otu }\end{array}$ & Aerial part & Chewed & (Gençay 2007) \\
\hline $\begin{array}{l}\text { Thymbra spicata } \\
\text { L. var. spicata }\end{array}$ & Lamiaceae & $\begin{array}{l}\text { Kara kekik, } \\
\text { Zahter, Kırçayı, } \\
\text { Bayır kekiği, } \\
\text { Karabaș otu, } \\
\text { Șeker otu }\end{array}$ & Aerial part & Inf. & (Balos and Akan 2007) \\
\hline
\end{tabular}


Table 1. The plants used in traditional toothache treatments in Turkey (Continued)

\begin{tabular}{|c|c|c|c|c|c|}
\hline $\begin{array}{l}\text { Botanical } \\
\text { name }\end{array}$ & Family & Local name & Plant part used & $\begin{array}{l}\text { Preparation, } \\
\text { and use }\end{array}$ & Ref. \\
\hline $\begin{array}{l}\text { Thymus longicaulis } \\
\text { subsp. chaubardii } \\
\text { (Rihb.f.) Jalas }\end{array}$ & Lamiaceae & $\begin{array}{l}\text { Kekik, Akbaşlı ot, } \\
\text { Güve otu, } \\
\text { Yer kekiği }\end{array}$ & Aerial part & $\begin{array}{l}\text { Chewed, Ext. } \\
\text { Oil, Ext. }\end{array}$ & (Özdemir Nath 2016) \\
\hline $\begin{array}{l}\text { T. zygoides Griseb. } \\
\text { var. zygoides }\end{array}$ & Lamiaceae & $\begin{array}{l}\text { Kekik, Dağ kekiği, } \\
\text { Bayır çayı, } \\
\text { Kaya kekiği, } \\
\text { Șeker otu, } \\
\text { Taș kekiği }\end{array}$ & Leaf and Seed & Chewed, Ext. & (Özdemir Nath 2016) \\
\hline $\begin{array}{l}\text { Tilia argentea } \\
\text { Desf. ex. DC. }\end{array}$ & Tiliaceae & Ihlamur & Flower & Inf., Mouthwash & (Akalın 1998) \\
\hline $\begin{array}{l}\text { Viscum album } \\
\text { L. subsp. album }\end{array}$ & Loranthaceae & $\begin{array}{l}\text { Ökse otu, } \\
\text { Büvelek otu, Burç, } \\
\text { Buruç, Güvelek }\end{array}$ & Aerial part & Dec., Mouthwash & (Sargın et al. 2013) \\
\hline $\begin{array}{l}\text { Xeranthemum } \\
\text { annuum L. }\end{array}$ & Asteraceae & $\begin{array}{l}\text { Süpürge otu, } \\
\text { Dağ karanfili, } \\
\text { Tarak çiçeği }\end{array}$ & Leaf & $\begin{array}{l}\text { +Tobacco, Like a } \\
\text { cigarette, Ext. }\end{array}$ & $\begin{array}{l}\text { (Tuzlacı and Doğan 2010; } \\
\text { Korkmaz and } \\
\text { Alpaslan 2014) }\end{array}$ \\
\hline
\end{tabular}

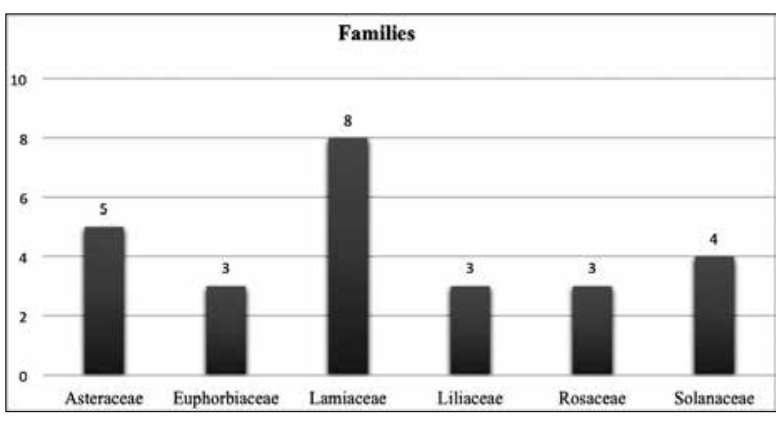

Figure 1. Graph of main families used in traditional toothache treatment in Turkey.

We hope that this study will contribute to the development of new medicines used in the treatment of toothache.

\section{References}

- Akalın E (1998). The plants of Tekirdag region that are used as medicinal purposes and food. J Tradit Folk Drugs 5(1): 1-96.

- Akan H, Korkut MM, Balos MM (2008) Arat Dağı ve çevresinde (Birecik, Şanlıurfa) etnobotanik bir araştırma. Science and Eng J of Firat Univ 20 (1): 67-81

- AktanT (2011). The Ethnobotanical examine in the villages of Yenisehir (Bursa). Unpublished MSc Thesis, Celal Bayar University, Manisa.

- $\quad$ Altundağ E, Öztürk M (2011). Ethnomedicinal studies on the plant resources of east Anatolia Turkey, Procedia Soc Behav Sci 19: 756-777. [CrossRef]

- Balos MM, Akan H (2007). Zeytinbahçe - Akarçay (Birecik, Şanlıurfa) arasında kalan bölgenin etnobotanik özellikleri. Selçuk Üniversitesi Fen Fakültesi Fen Dergisi 29: 155-171.

- Baytop T (1971). Farmakognozi Ders Kitabı Cilt 2. Baha Matbaası, Istanbul.

- Beguma S, Saxenaa B, Goyala M, Ranjanb R, Joshia VB, Raoc CV, Krishnamurthya S, Sahaib M (2010). Study of anti-inflammatory, analgesic and antipyretic activities of seeds of Hyoscyamus niger and isolation of a new coumarinolignan. Fitoterapia 81(3): 178184. [CrossRef]

- Bulut G, Tuzlacı E (2009). Folk medicinal plants of Bayramiç (Çanakkale-Turkey). J Fac Pharm Istanbul 40: 87-100.
Dindaa B, Kyriakopoulosb AM, Dindac S, Zoumpourlisd V, Thomaidise NS, Velegrakif A, Markopoulosg C, Dindah M (2016). Cornus mas L. (cornelian cherry), an important European and Asian traditional food and medicine: Ethnomedicine, phytochemistry and pharmacology for its commercial utilization in drug industry. J Ethnopharmacol 193: 670-690. [CrossRef]

- Ecevit Genç G, Özhatay N (2006). An ethnobotanical study in Çatalca (European part of Istanbul) II. Turkish J Pharm Sci 3 (2): 73-89.

- Electronic resource 1. İstanbul Ağrı Merkezi. www.agritr.com/ html/algoloji.html Accessed: 14.04.2017.

- Electronic resource 2. Diş Ağrılarının Nedenleri ve Çözümleri. https://www.hospitadent.com/dis-sagligi/dis-agrilarinin-nedenleri-ve-cozumleri.html Accessed: 14.04.2017.

- $\quad$ Erbay MŞ, Anıl S, Melikoğlu G (2017). Plants used as painkiller in folk medicine in Turkey-l Stomachache. J Res Pharm 21 (4): 741-755. Erbay MŞ, Anıl S, Melikoğlu G (2018a). Plants used as painkiller in folk medicine in Turkey-II Headache. J Res Pharm 22(1): 2936.

- $\quad$ Erbay MŞ, Anıl S, Melikoğlu G (2018b). Plants used as painkiller in folk medicine in Turkey-III Rheumatic pain. J Res Pharm 22(2): 133-141. [CrossRef]

- Erdoğan R (2011). Ethnobotanical features some of the wild on the Sariveliler (Karaman) and its environment ethnobotanic. Unpublished MSc Thesis, Selcuk University, Konya.

- $\quad$ Eşen B (2008). The ethnobotanical properties of Aydınlar village and its vicinity (Erdemli/Mersin). Unpublished MSc Thesis, Selcuk University, Konya.

- Fujita T, Sezik E, Tabata M, Yeşilada E, Honda G, Takeda Y, Tanaka T, Takaishi Y (1995). Traditional medicine in Turkey VII. Folk medicine and west Black Sea regions. Econ Bot 49(7): 406-422. [CrossRef]

- Gençay A (2007). Ethnobotanical aspects of Cizre (Sirnak). Unpublished MSc Thesis, Yuzuncu Yil University, Van.

- Güner A, Özhatay N, Ekim T, Başer KHC (2000). Flora of Turkey and the East Islands. vol. 11. Edinburgh University Press, Edinburgh.

- Güneş S, Savran A, Paksoy MY, Koşar M, Çakılcıoğlu U (2017). Ethnopharmacological survey of medicinal plants in Karaisalı and its surrounding (Adana-Turkey). J Herb Med 8: 68-75. [CrossRef]

Kargıoğlu M, Cenkci S, Serteser A, Konuk M, Vural G (2010). Traditional uses of wild plants in the Middle Aegean Region of Turkey. Hum Ecol. 38: 429-450. [CrossRef] 
- Korkmaz M, Alpaslan Z (2014). Ergan Dağı (Erzincan-Türkiye)'nın etnobotanik özellikleri. Bağbahçe Bilim Dergisi 1(3): 1-31.

- Kültür \$̧ (2007). Medicinal plants used in Kirklareli province (Turkey). J Ethnopharmaco/ 111: 341-364. [CrossRef]

- Metin A (2009). Ethnobotanical features of plants in Mut (Mersin) and its environments. Unpublished MSc Thesis, Selcuk University, Konya.

- Mükemre M, Behçet L, Çakılcıoğlu U (2015). Ethnobotanical study on medicinal plants in villages of Çatak (Van-Turkey). J Ethnopharmacol 166: 361-374. https://doi.org/10.1016/j.jep.2015.03.040 [CrossRef]

- Oral ÇD (2007). Ethnobotanical studies on folk medicines used in Konya. Unpublished MSc Thesis, Gazi University, Ankara.

- Özdemir E, Alpınar K (2015). An ethnobotanical survey of medicinal plants in western part of central Taurus Mountains: Aladaglar (Nigde- Turkey). J Ethnopharmacol 166: 53-65. [CrossRef]

- Özdemir Nath E (2016). An Ethnobotanical Study in Savastepe and Kepsut Region (Balikesir). Unpublished PhD Thesis, Istanbul University, Istanbul.

- Özgen U, Kaya Y, Houghton P (2012). Folk medicines in the villages of llica district (Erzurum, Turkey). Turk J Bio/ 36: 93-106.

- Özhatay N, Kültür \$̧, Gürdal B (2013). Check-list of Additional Taxa to the Supplement Flora of Turkey VI. J Fac Pharm Istanbul $\mathbf{4 3 ( 1 )}$ 33-82.

- Özhatay N, Kültür Ş, Gürdal B (2015). Check-list of Additional Taxa to the Supplement Flora of Turkey VII. J Fac Pharm Istanbul 45 (1): 61-86.

- Polat R, Satıl F (2012). An ethnobotanical survey of medicinal plants in Edremit Gulf (Balıkesir - Turkey). J Ethnopharmacol 139 : 626-641. [CrossRef]

- $\quad$ Renn CL, Dorsey SG (2005). The physiology and processing of pain a review. AACN Adv Crit Care 16.3: 277-290.

- $\quad$ Saday H (2009). Ethnobotanical properties of Güzeloluk village and its vicinity. Unpublished MSc Thesis, Selcuk University, Konya.

- Sadıkoğlu N, Alpınar K (2001). Etnobotanik açıdan Bartın. In: Gürkan E, Tuzlacı E (Eds.) 13. Bitkisel Ilaç Hammaddeleri Toplantısı Bildiri Kitabı. M.Ü.Ecz. Fak. Yay. No: 17, pp.87. Istanbul, Turkey.

- Sağıroğlu M, Arslantürk A, Akdemir ZK, Turna M (2012). An ethnobotanical from Hayrat (Trabzon) and Kalkandere (Rize/Turkey) Bio DiCon 5/1: 31-43.
Şahin Yiğit S (2014). Medicinal plants sold in Gaziantep herbalists and their ethnobotanical aspects. Unpublished MSc Thesis, Gaziantep University, Gaziantep.

- Saraç DU, Özkan ZC, Akbulut S (2013). Ethnobotanic features of Rize/Turkey province. Bio DiCon 6/3: 57-66.

- Sargın SA, Akçiçek E, Selvi S (2013). An ethnobotanical study of medicinal plants used by the local people of Alaşehir (Manisa) in Turkey. J Ethnopharmacol 150 (3): 860-874. https://doi. org/10.1016/j.jep.2013.09.040 [CrossRef]

Şenkardeş I (2014). Ethnobotanical investigations in southern districts (Acigöl, Derinkuyu, Gulsehir, Nevsehir-Central district, Urgup) of Nevsehir. Unpublished PhD Thesis, Marmara University, Istanbul.

- Sezik E, Yeşilada E, Tabata M, Honda G, Takaishi Y, Fijita T, Tanaka T, Takeda Y (1997). Traditional medicine in Turkey VIII. Folk medicine in East Anatolia; Erzurum, Erzincan, Agri, Kars, Igdir provinces. Econ Bot 51(6): 195-211. [CrossRef]

- Tetik F, Civelek S, Cakilcioglu U (2013). Traditional uses of some medicinal plants in Malatya (Turkey). J Ethnopharmacol 146: 331346. [CrossRef]

- Tuzlacı E, Alparslan DF (2007). Turkish folk medicinal plants, Part V: Babaeski (Kirklareli), J Fac Pharm Istanbul 39: 11-23.

- Tuzlacı E, Doğan A (2010). Turkish folk medicinal plants, IX: Ovacık (Tunceli). J Res Pharm 14: 136-143. [CrossRef]

Tuzlacı E, Eryaşar Aymaz P (2001). Turkish folk medicinal plants, Part IV: Gonen (Balikesir). Fitoterapia. 72: 323-343. [CrossRef]

- Tuzlacı E, Sadıkoğlu E (2007). Turkish folk medicinal plants, Part Vl: Koçarlı (Aydın). J Fac Pharm Istanbul 39: 25-38.

Tuzlacı E, Tolon E (2000). Turkish folk medicinal plants. Part III: Sile (Istanbul). Fitoterapia 71: 673-685. [CrossRef]

Ugulu I (2011). Traditional ethnobotanical knowledge about medicinal plants used for external therapies in Alasehir, Turkey. Int J Med Arom Plants 1(2): 101-106.

- Vural G (2008). Ethnobotanical features some of the willd plants on the Honaz mountain and its environment ethnobotanic. Unpublished MSc Thesis, Afyon Kocatepe University, Afyon.

- Yatani H, Komiyama O, Matsuka Y, Wajima K, Muraoka W, Ikawa M, Sakamoto E, De Laat A, Heir GM (2014). Systematic review and recommendations for nonodontogenic toothache. J Oral Rehabil 41(11): 843-852. [CrossRef] 